\title{
Preparation of Simvastatin Hydrogel through Arginine Addition for Drug Delivery System
}

\author{
Niswati Fathmah Rosyida ${ }^{1}$, Teguh Ariyanto ${ }^{2 *}$, Pinandi Sri Pudyani ${ }^{1}$, Ika Dewi Ana $^{3 *}$ \\ ${ }^{1}$ Department of Orthodontic, Faculty of Dentistry, Universitas Gadjah Mada, 55281, Yogyakarta, Indonesia \\ ${ }^{2}$ Department of Chemical Engineering, Faculty of Engineering, Universitas Gadjah Mada, 55281, Yogyakarta, Indonesia \\ ${ }^{3}$ Department of Biomedicine, Faculty of Dentistry, Universitas Gadjah Mada, 55281, Yogyakarta, Indonesia
}

\begin{abstract}
Simvastatin is a lipid lowering agent which has been used recently as drug delivery system for stimulating bone regeneration. Because of low therapeutic efficacy and bioavailability, it is necessary to deliver simvastatin by local administration e.g. by hydrogel system. However, simvastatin has very poor solubility which restricts to prepare hydrogel formulation. The aim of this study is to improve solubility of simvastatin with arginine as co-solvent for developing a controlled released drug delivery system by loading simvastatin into gelatin hydrogel. The solubility study was performed by addition of an excess mass of simvastatin into the specified molar solutions of the arginine. All conical flasks were placed in a mechanical water bath shaker at the temperature of 25,40 , and $50^{\circ} \mathrm{C}$ and shaken for a maximum period of 72 hours. The drug concentration was analyzed by UV/Visible spectroscopy at $238 \mathrm{~nm}$. The hydrogel was prepared by a dehydrothermal method. The results showed that simvastatin solubility increases with increasing arginine concentrations and temperature. Characterizations showed a successful preparation of simvastatin-loaded gelatin hydrogel. The arginine simvastatin hydrogel and the gelatin hydrogel (as a blank) exhibited a comparable swelling index (ca. 6.5). Furthermore, microparticles of the material show a narrow particle size distribution in the range between 150$250 \mu \mathrm{m}$.
\end{abstract}

\section{Introduction}

Bone damage due to trauma, osteoporosis, and genetic diseases is a health issue that requires serious treatment. Bone injury can decrease the quality of life, so is needed therapy to accelerate the bone healing process. Without the aid of drugs and bone substitution, healing of bones takes a long time and is less than perfect [1]. Recently, one of the drugs known to have effects on bone healing is simvastatin. Simvastatin is a drug used to lower cholesterol in the blood through inhibition of cholesterol-forming enzymes [2]. Simvastatin could induce bone formation by increasing bone morphogenetic protein expression (BMP-2) and inhibiting bone resorption by reducing RANKL expression [3,4]. However, the bioavailability of simvastatin is only about $5 \%$, since it is metabolized in the liver [2]. Systemic administration of high doses of simvastatin is required to obtain statin effects on bone, but increased doses will also enhance the toxicity of a drug [5]. Therefore, it is necessary to modify the simvastatin formulas given either injections or implants locally to obtain the optimal therapeutic effect on bone.

The hydrogel is a drug carrier polymer that is currently developed due to many advantages. The natural material employed to make the hydrogel is gelatin, which has a biocompatible, and can be degraded [6]. The combination of active substances of simvastatin and gelatin is expected to become the right combination formula for bone healing therapy with local injection. Arginine is one type of amino acid that can be used to improve drug solubility [7]. Furthermore, arginine can accelerate wound healing [8]. Arginine has antibacterial effect and promotes collagen synthesis and cell proliferation [9].

The aim of this study is to improve solubility of simvastatin with arginine as co-solvent for developing a controlled drug delivery system by loading simvastatin into gelatin hydrogel. Material preparation and characterization of drug-loaded hydrogel are also discussed.

\section{Material and methods}

\subsection{Materials}

Simvastatin and arginine (pharmaceutical grade, Sigma Aldrich, Singapore) were used as active substance and

Corresponding author: teguh.ariyanto@ugm.ac.id; ikadewiana@ugm.ac.id 
surfactant, respectively. Gelatin type B was obtained from Nitta Osaka, Japan.

\subsection{Solubility study of simvastatin}

Surfactants in the $0.01-1 \mathrm{~mol} \mathrm{~L}^{-1}$ concentration range were prepared by weighing the right material mass and incorporated into $100 \mathrm{~mL}$ of water in Erlenmeyer. The mixture was then stirred to form a homogeneous solution. Excessive amounts of simvastatin were added to each Erlenmeyer containing the specified molar solutions of the arginine. All conical flasks were placed in an incubator shaker under controlled temperature conditions $\left(25^{\circ} \mathrm{C}, 40{ }^{\circ} \mathrm{C}\right.$, and $\left.50{ }^{\circ} \mathrm{C}\right)$ up to a maximum of $72 \mathrm{~h}$ to ensure equilibrium. At the end of the incubation period, the suspensions were filtered and measured its absorbance by UV/Vis spectroscopy at position $238 \mathrm{~nm}$. The typical UV/Vis spectra of simvastatin solution are shown in Fig. 1. A linier correlation of absorbance vs. simvastatin concentration results.

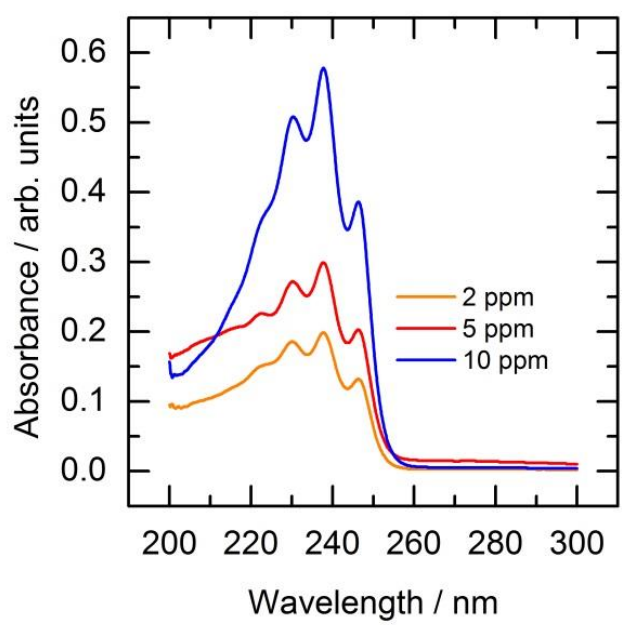

Fig. 1. UV/Vis spectra of simvastatin solution showing a maximum peak at $238 \mathrm{~nm}$.

\subsection{Preparation of microparticle of simvastatin- arginine-gelatin hydrogel}

Hydrogels are made from gelatin with $7 \%$ w/v concentration. Gelatin powder was dissolved in $10 \mathrm{ml}$ of distilled water in a beaker and waited until it was swelling. Gelatin was then stirred on a water bath until homogeneous solution. Simvastatin and arginine were added to gelatin solution and stirred at $40^{\circ} \mathrm{C}$. The mixture was cast into polypropylene mold. Specimens were then included in the freeze dryer overnight. Subsequently, the sample was wrapped in parchment paper and aluminum foil and inserted into a vacuum oven until obtaining solid material. The formed hydrogel block matrix was then crushed to obtain microparticles.

\subsection{Characterization of simvastatin-arginine- gelatin hydrogel}

Image of microparticles was taken by stereomicroscope (Olympus SZX7, Japan). Swelling of the hydrogel index was measured through a hydration process. First, the initial weight of the hydrogel was measured by an electric balance. The hydrogel was then immersed in $100 \mu \mathrm{L}$ of aquadest. The final weight of the hydrogel was measured at every hour for seven hours. The swelling index was defined as an increased mass after immersion divided by the initial mass of hydrogel.

The Fourier Transform Infrared Spectroscopy (FTIR) test was performed to determine the functional and qualitative groups of hydrogel microparticles and was taken by NICOLET iS10 (Thermoscientific, USA). The sample was mixed with $\mathrm{KBr}$ and compacted on the disc. The translucent film was scanned over a range of 4000 to $400 \mathrm{~cm}^{-1}$ at ambient temperature.

\section{Results and discussion}

\subsection{Solubility of simvastatin in arginine solution}

To investigate the amount of simvastatin that can be dissolved in water solution as solvent in hydrogel preparation, solubility study was carried out by introducing arginine in different concentrations. Fig. 2 displays solubility of simvastatin as a function of arginine concentration and temperature. The solubility of simvastatin in water is ca. $0.01 \mathrm{mmol} \mathrm{L}^{-1}$ at room temperature. This result is in agreement with other studies of simvastatin solubility in pure water [10]. When a higher temperature is used $\left(40^{\circ} \mathrm{C}\right)$, the solubility is a little bit increased. At room temperature, as can be seen, the solubility increases linearly when introducing arginine. At $0.1 \mathrm{M}$ arginine, solubility of simvastatin can be enhanced seven times higher than that of in water. The enhancement of solubility can further be increased at higher solution temperature of $40^{\circ} \mathrm{C}$. However, the study showed that increasing further the temperature to $50{ }^{\circ} \mathrm{C}$ does not give a positive impact on solubility of simvastatin. This is due to the arginine is not stable at a high temperature [11]. 


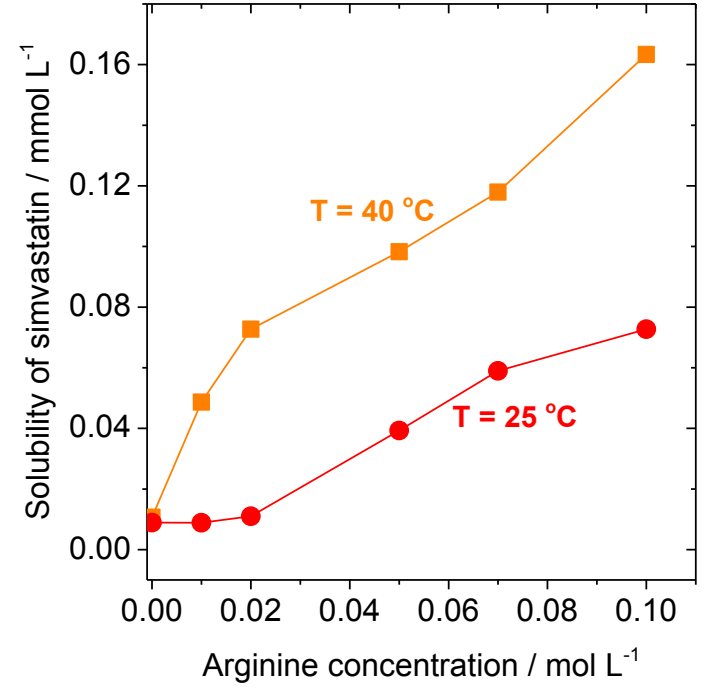

Fig. 2. Solubility of simvastatin as a function of temperature and arginine concentration $(\mathrm{pH}=7)$.

The positive impact of arginine in solubility of simvastatin could give an advantage when dispersing simvastatin as an active substance in hydrogel media.

\subsection{Hydrogel microparticle synthesis and characterizations}

The active substance of simvastatin was dispersed in hydrogel carrier. After size reduction of hydrogel which contains arginine-simvastatin, particle sizes were observed by using stereomicroscope. Fig. 3 shows microparticles with a relatively homogenous size ca. $200 \mu \mathrm{m}$. This evidences that microparticles are obtained. The hydrogel exhibited the swelling index of more than 4 which indicates the material can adsorb high amount of water.

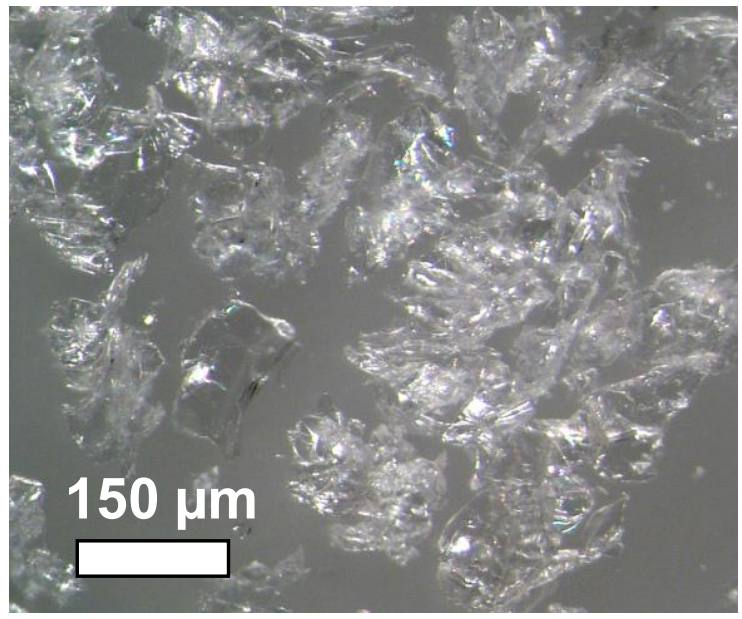

Fig. 3. Hydrogel microparticles observed by stereomicroscope.
Fig. 4 shows the swelling index of gelatin arginine simvastatin and pure gelatin hydrogel. Simvastatin hydrogel showed the higher swelling rather than the pure gelatin hydrogel. Swelling index is influenced by the composition and the degree of cross linking [12]. Swelling index is lower when the degree of cross linking is high. In the present study, there was no significant difference between hydrogel with or without simvastatin.

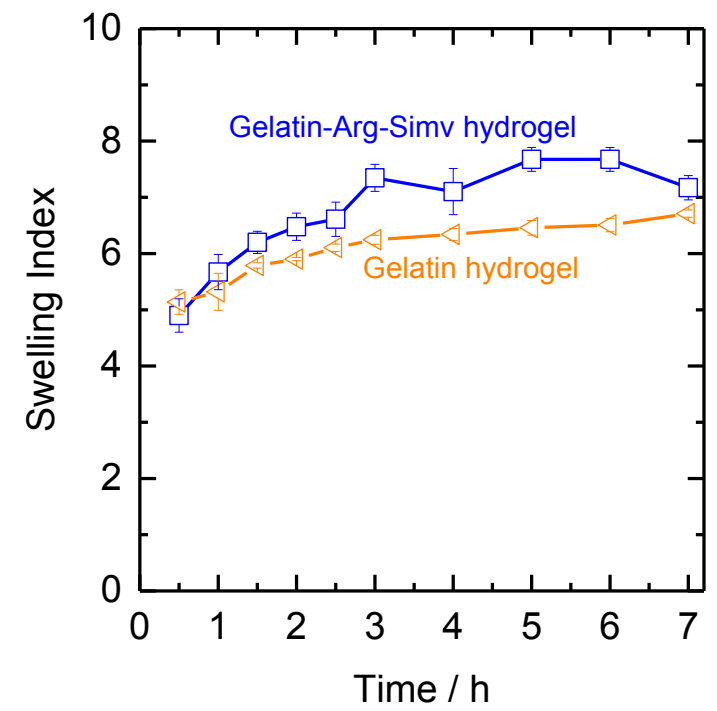

Fig. 4. Swelling index of hydrogel.

To further study the success of preparation of simvastatin-arginine-gelatin hydrogel and functional group involved in the material, FTIR study was carried out. FTIR profiles for gelatin, arginine, simvastatin, and the hydrogel are shown in Fig. 4. As individual substances, simvastatin exhibits clearly hydroxyl $(-\mathrm{OH})$ at ca. $3550 \mathrm{~cm}^{-1}$ peak, alkyl (-RH) at ca. $3000 \mathrm{~cm}^{-1}$ peak and alkene $(\mathrm{C}=\mathrm{C})$ at ca. $1950 \mathrm{~cm}^{-1}$ peak of functional groups. For arginine and gelatin which are amino acid and protein, it can be seen - $\mathrm{NH}$ functional group in the range of $3200-3500 \mathrm{~cm}^{-1}$ wavelength. After all of the substances were mixed in the hydrogel system, dominantly of gelatin signal is observed. But a stronger FTIR spectrum is seen. This is likely due to a presence 
of simvastatin and arginine in hydrogel system.

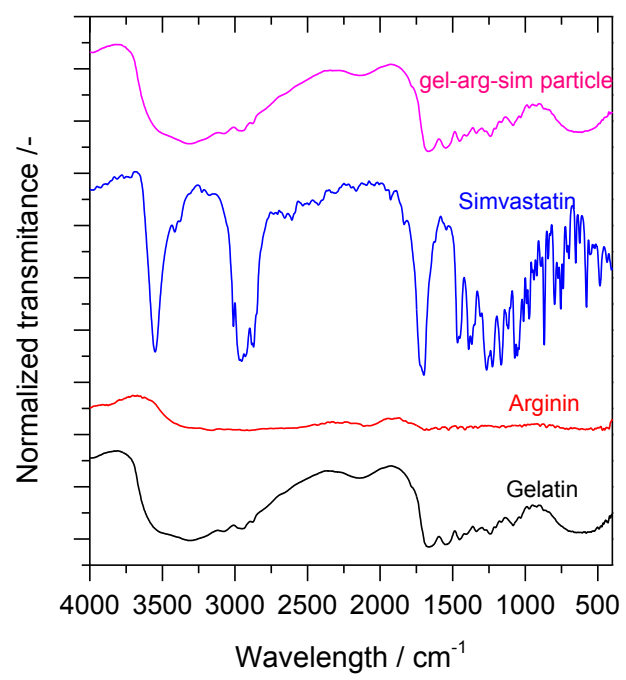

Fig. 4. FTIR signals of hydrogel microparticle and its components.

\section{Conclusions}

The solubility of simvastatin can be enhanced by addition of arginine in water. The study showed that concentration of arginine and temperature of solution have significant effects on the simvastatin solubility. This positive influence of arginine was further used to formulate simvastatin-loaded hydrogel based on gelatin. Characterizations showed a successful preparation of hydrogel microparticles.

The authors gratefully acknowledge the funding of the Ministry of Research, Technology and Higher Education of the Republic of Indonesia (MENRISTEKDIKTI) through Penelitian Fundamental 2017 (Grant No. 7183/UN1.P.III/DIT-LIT/LT/2017). NFR thanks to Universitas Gadjah Mada for funding of Hibah Peningkatan Kapasitas Dosen Muda 2017.

\section{References}

1. Y. Kim, Y. Tabata, Adv. Drug Delivery Rev. 94, 28-40 (2015)

2. CR. Sirtori, Pharmacol Res. 88, 3-11 (2014)

3. Z. Wu, C. Liu, G. Zang, H. Sun, Int. J. Oral Maxillofac. Surg, 37, 170-6 (2008)

4. I. R. Garret, G. R. Mundy, Arthritis Res. 4, 237-40 (2002)

5. Z. Jia, et al, J. Controlled Release. 200, 23-34 (2015)

6. J. Zhang, H. Wang, Y. Wang, K. Lai, X. Yang, X. Chen, G. Yang, Scientific Report. 158, 1-12 (2016)

7. A. Hirano, T. Kameda, T. Arakawa, K. Shiraki, J. Phys. Chem. B. 114, 13455-62 (2010)

8. S. Sinha, S. C. Goel, Indian J of Orthop, 43, 328-34 (2009)
9. M. He, A. Potuck, Y. Zhang, CC. Chu, Acta Biomater, 10, 2482-94 (2014)

10. M. Rúbia, W De. Vargas, FN. Raffin, T. Flávio, A De. Lima, Rev Ciênc Farm Básica Apl, 33, 497-507 (2012)

11. G. Wu, Amino Acids: Biochemistry and Nutrition (CRC Press, 2013)

12. S. Yasasvini, R. Anusa, B. Vedhahari, P. Prabhu, D. Ramyadevi, Mater Sci Eng C, 72, 160-7 (2017) 